\section{Cureus}

\title{
Concurrent Occurrence of Primary Biliary Cirrhosis and Rheumatoid Arthritis
}

\author{
Stella Pak ${ }^{1}$, Umar Darr ${ }^{1}$, Zubair Khan ${ }^{1}$, Andrew Kobalka ${ }^{2}$, Zayd Safadi ${ }^{3}$, Christine Dee ${ }^{4}$ \\ 1. Internal Medicine, University of Toledo Medical Center 2. Department of Pathology, University of \\ Toledo Medical Center 3. College of Medicine and Life Sciences, University of Toledo Medical Center 4. \\ Wright State University Boonshoft School of Medicine
}

$\square$ Corresponding author: Stella Pak, stella.pak@rockets.utoledo.edu

Disclosures can be found in Additional Information at the end of the article

\section{Abstract}

Primary biliary cirrhosis (PBC) is an autoimmune cholestatic disorder of the liver. A diagnostic serum marker for PBC is an anti-mitochondrial antibody. Most prominent histologic findings of $\mathrm{PBC}$ are portal inflammation and destruction of interlobular bile ducts. The PBC occurs only in 40 to 400 individuals per million in the general population. About $1.8-5.6 \%$ of individuals with this rare disorder have rheumatoid arthritis (RA). This case report describes a 56-year-old female with concurrent rheumatoid arthritis and primary biliary cirrhosis. The patients with RA are at higher risk of developing $\mathrm{PBC}$ compared to the general population. Thus, abnormal liver function test in the patients with RA, especially in the absence of alternative cause, warrants thorough investigation for PBC. Early diagnosis and treatment will improve the outcome of patients who develop PBC.

Categories: Internal Medicine

Keywords: rheumatoid arthritis, primary biliary cirrhosis

\section{Introduction}

Rheumatoid arthritis (RA) is an autoimmune inflammatory disorder characterized by synovitis. The prevalence of RA is estimated to be about $0.5-1 \%$ of the general population. In approximately two-third of individuals with RA, various types of liver histological abnormalities are found, including periportal fibrosis, portal tract inflammation, and sinusoidal dilatation. The lack of piecemeal and bridging necrosis or plasma cell infiltration are hallmarks of RA-associated liver disease [1]. The abnormalities, however, tend to be mild and have no clinical manifestation.

Received 07/19/2017 Review began 08/08/2017 Review ended 08/08/2017 Published 08/13/2017

\section{C) Copyright 2017}

Pak et al. This is an open access article distributed under the terms of the Creative Commons Attribution License CC-BY 3.0., which permits unrestricted use, distribution, and reproduction in any medium, provided the original author and source are credited.
Primary biliary cirrhosis (PBC) is an autoimmune cholestatic disorder of the liver. A diagnostic serum marker for $\mathrm{PBC}$ is an anti-mitochondrial antibody. Most prominent histologic findings of PBC are portal inflammation and destruction of interlobular bile ducts. The PBC occurs only in 40 to 400 individuals per million in the general population [2]. About $1.8-5.6 \%$ of individuals with this rare disorder have RA [3]. This concurrent manifestation of two rare diseases implies their pathophysiological association. Informed consent was obtained from the patient for this study.

\section{Case Presentation}

A 56-year-old female with an 11-year history of RA was found to have mild elevations of aspartate aminotransferase (AST) of $54 \mathrm{U} / \mathrm{L}$ [normal values $=25 \mathrm{U} / \mathrm{L}]$ and alanine aminotransferase (ALT) of $49 \mathrm{U} / \mathrm{L}$ [normal values 


\section{Cureus}

$=27 \mathrm{U} / \mathrm{L}]$ without evidence of hepatomegaly or ascites. Serologic testing revealed a 1:640 anti-mitochondrial antibody titer consistent with PBC. Hepatitis panel was negative. Liver biopsy showed increased collagen in portal areas with portal widening (Figure 1), mild piecemeal necrosis and a mixed inflammatory infiltrate (Figure 2). Hepatic ultrasound visualized mild heterogeneous increase in echogenicity of the liver, consistent with mild fibrotic change (Figure 3).

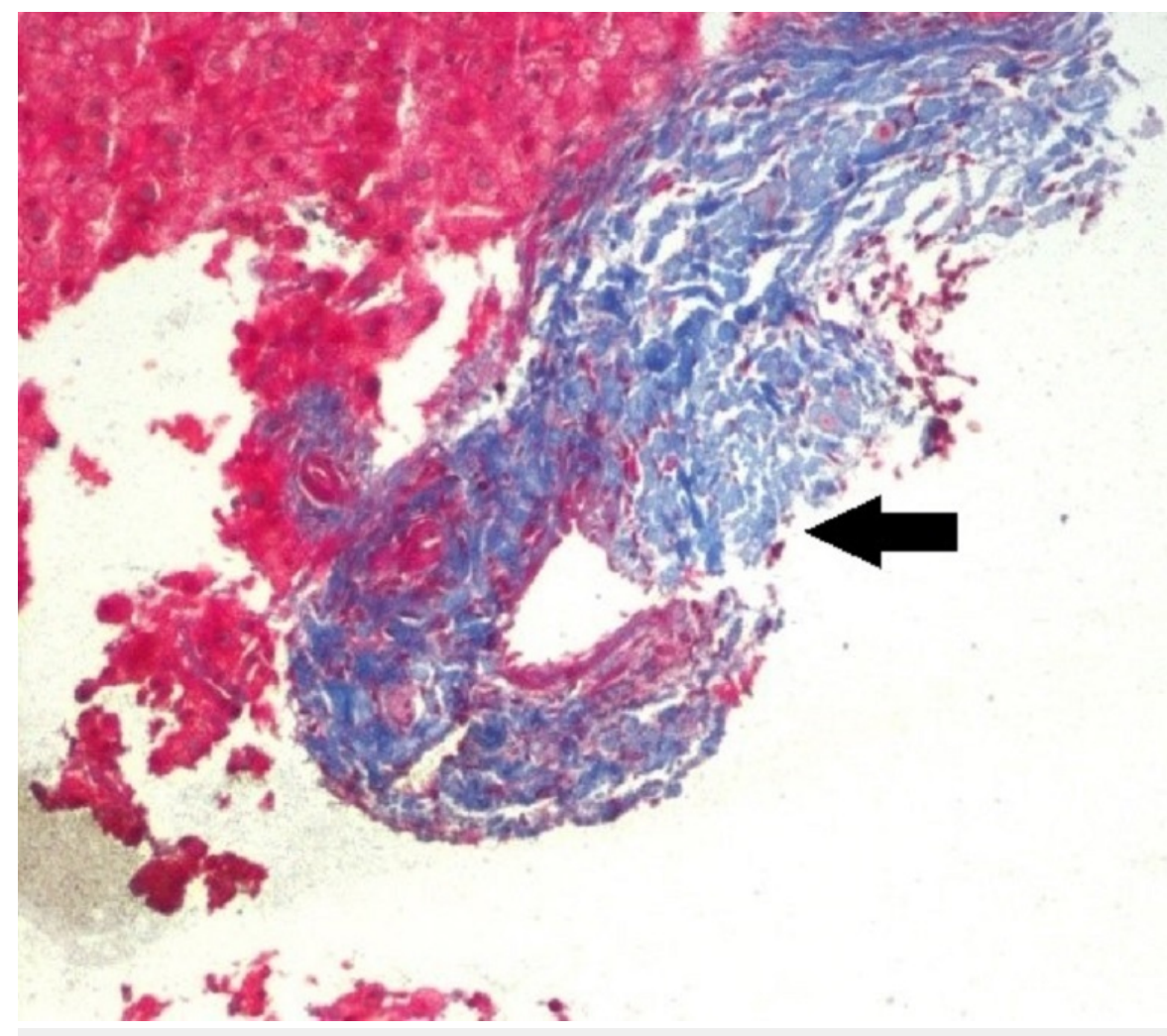

FIGURE 1: Liver biopsy showing increased collagen (black arrow) in portal areas with fibrous widening 


\section{Cureus}

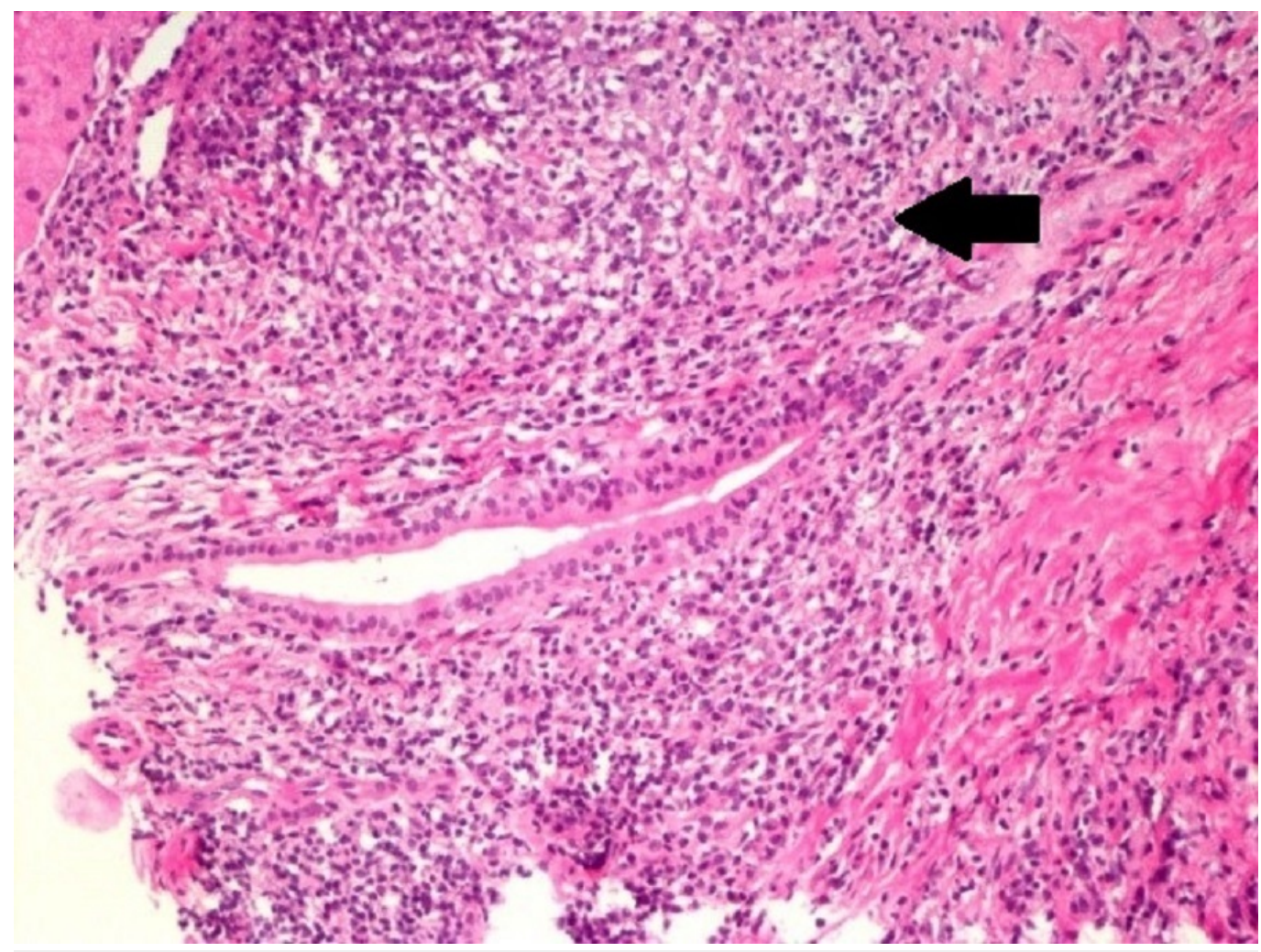

FIGURE 2: Mixed inflammatory infiltrate (black arrow) in portal areas with increased collagen and piecemeal necrosis

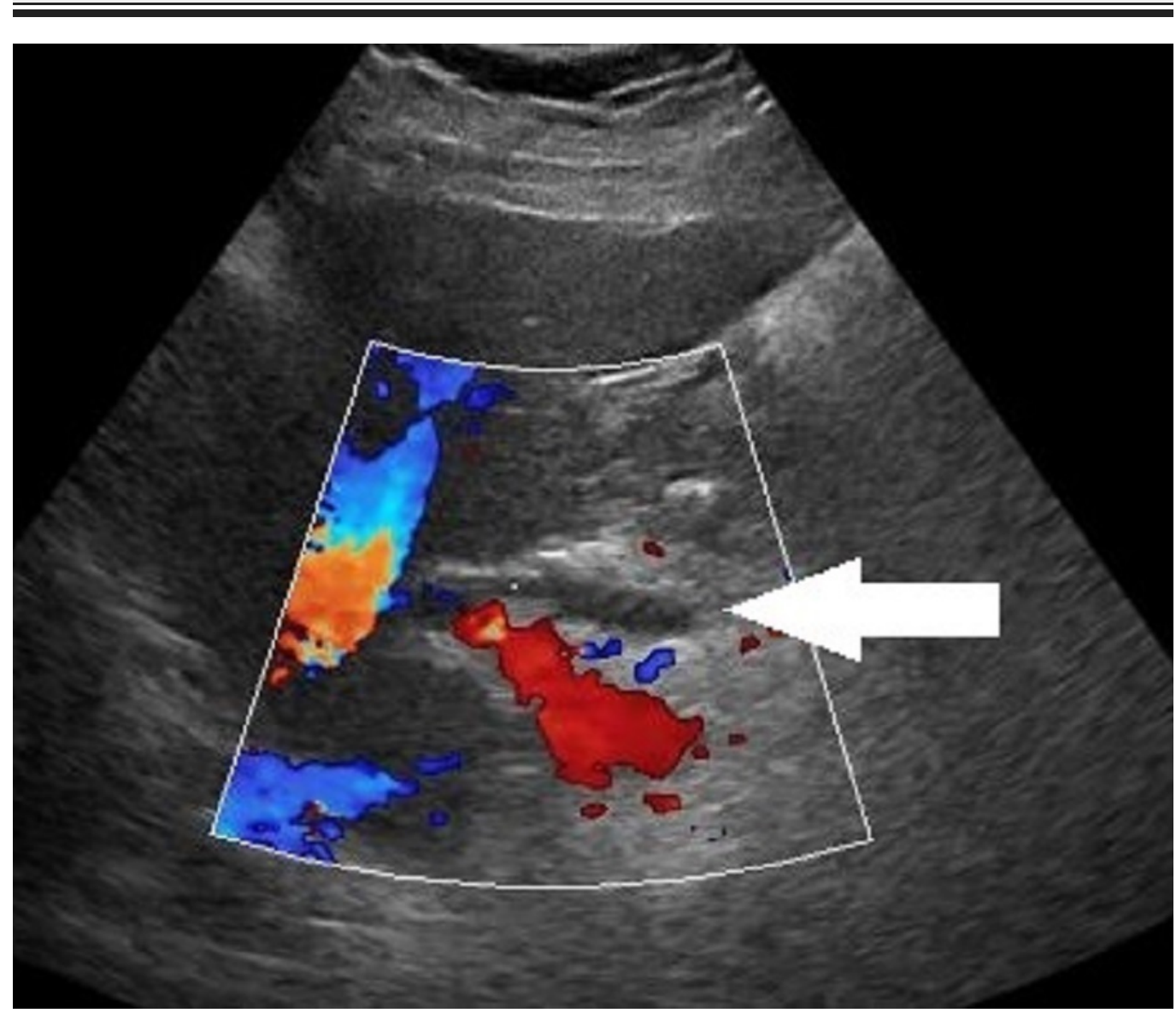


FIGURE 3: Hepatic ultrasound visualized mild heterogeneous increase (white arrow) in echogenicity of the liver, consistent with mild fibrotic change

Ursodeoxycholic acid $250 \mathrm{mg}$ twice daily was prescribed to treat the patients' $\mathrm{PBC}$. While clinically asymptomatic over six months, the patients' liver function tests remained elevated (AST 36 U/L, ALT 43 $\mathrm{U} / \mathrm{L}$ ) due to prompted uptitration of ursodeoxycholic acid to $500 \mathrm{mg}$ twice daily and as a result reducing her AST and ALT to normal limits.

\section{Discussion}

The concurrent occurrence of RA and PBC in our patient supports the possible association between these two rare clinical entities. A postulated hypothesis for this association is genetic locus homogeneity, with the following genes found to be implicated in both disorders: histocompatibility complex, class II, DQ beta 1 ( HLA-DQB1), cytotoxic T-lymphocyte-associated protein 4 (CTLA4), membrane metallo-endopeptidase-like 1 (MMEL1), interferon regulatory factor 5 (IRF5), Signal transducer and activator of transcription 4 (STAT4) and CXC chemokine receptor 5 ( CXCR5) [4].

About $50-60 \%$ of individuals with PBC are asymptomatic at the time of diagnosis. However, if left untreated, they go on to develop symptoms such as pruritus, fatigue, and ascites [5]. It is reported that most patients with concurrent RA and $\mathrm{PBC}$ tend to develop RA years before PBC [6]. Being aware of the potential link between RA and PBC, it may be clinically warranted to evaluate RA patients with abnormal liver function test for $\mathrm{PBC}$, even if they are asymptomatic.

\section{Conclusions}

The patients with RA are at higher risk of developing PBC compared to the general population. Thus, abnormal liver function test in the patients with RA, especially in the absence of alternative cause, warrants thorough investigation for PBC. Early diagnosis and treatment will improve the outcome of patients who develop PBC.

\section{Additional Information}

\section{Disclosures}

Human subjects: Consent was obtained by all participants in this study. Conflicts of interest: In compliance with the ICMJE uniform disclosure form, all authors declare the following:

Payment/services info: All authors have declared that no financial support was received from any organization for the submitted work. Financial relationships: All authors have declared that they have no financial relationships at present or within the previous three years with any 


\section{Cureus}

organizations that might have an interest in the submitted work. Other relationships: All authors have declared that there are no other relationships or activities that could appear to have influenced the submitted work.

\section{References}

1. Ebert EC, Hagspiel KD: Gastrointestinal and hepatic manifestations of rheumatoid arthritis . Dig Dis Sci. 2011, 56:295-302. 10.1007/s10620-010-1508-7

2. Kim KA, Jeong SH: The diagnosis and treatment of primary biliary cirrhosis . Korean J Hepatol. 2011, 17:173-179. 10.3350/kjhep.2011.17.3.173

3. Smyk DS, Bogdanos DP, Mytilinaiou MG, et al.: Rheumatoid arthritis and primary biliary cirrhosis: Cause, consequence, or coincidence?. Arthritis. 2012, 2012:1-7. 10.1155/2012/391567

4. Bekki N, Bae S, Yoshizawa S, et al.: A case of primary biliary cirrhosis in a patient with rheumatoid arthritis. Clin Case Rep. 2016, 4:90-94. 10.1002/ccr3.449

5. Liu J, Zhou X, Han Z, et al.: Primary biliary cirrhosis associated with rheumatoid arthritis: two case reports in China. EXCLI Journal. 2007, 6:183-186.

6. Caramella C, Avouac J, Sogni P, et al.: Association between rheumatoid arthritis and primary biliary cirrhosis. Joint Bone Spine. 2007, 74:279-281. 10.1016/j.jbspin.2006.06.012 